\title{
GE/MCKINSEY MATRICES REVISITED: A MIXED MODE TOOL FOR MULTI-CRITERIA DECISION ANALYSIS
}

\author{
Mariza Tsakalerou \\ Department of Production Engineering \& Management, \\ Democritus University of Thrace, Xanthi, Greece
}

Doi: 10.19044/elp.v2no1a5 URL:http://dx.doi.org/10.19044/elp.v2no1a5

\begin{abstract}
Multi-criteria decision analysis is an umbrella term describing the collection of formal approaches that take explicit account of multiple criteria in order to explore alternative decisions. Multi-criteria analysis has evolved from its operations research origins to address situations where the criteria are not so easily quantified and where the data is often fuzzy. In this context, GE/McKinsey matrices, a classic tool of the business portfolio analysis, have emerged as a mixed-mode tool to cover modern problems with fuzzy data and simultaneous use of quantitative and qualitative variables. By grouping the variables into a small number of classes, GE/McKinsey matrices provide an effective way to reduce the effect of noise in the data and to identify only major trends thus strengthening the conclusions of the corresponding decision analysis.
\end{abstract}

Keyords: Multi-criteria decision analysis; Mixed-mode analysis; GE/McKinsey matrices

\section{Introduction}

Decision-making is regarded as the cognitive process resulting in the selection of a course of action among several alternative possibilities (Lu et al. 2007). While decision-making involves the analysis of a finite set of alternatives described in terms of evaluative criteria, the final choice is based on the values and preferences of the decision makers (Harris 1998). Realworld problems typically demand multiple perspectives from different stakeholders, and thus a decision is usually understood to be the reduction of multiple individual views into a single collective preference.

Deciding on which alternative to choose amidst conflicting multiple criteria and multiple perspectives of stakeholders is a challenging task. Decision theory coupled with operational research techniques has been 
traditionally used in practice to solve complex decision problems by ranking a finite number of decision options based a finite set of evaluation criteria (Lu et al. 2007).

The term multi-criteria decision analysis (MCDA) has been used to describe the analytical tools used to explore complex decision problems characterized by multiple (and possibly conflicting) objectives and criteria. The generally accepted definition of Belton and Stewart (2002) that MCDA is "an umbrella term to describe a collection of formal approaches which seek to take explicit account of multiple criteria in helping individuals or groups explore decisions that matter» reflects this use of the term.

Increasingly though MCDA is used as a term to signify the systematic framework employed to support the initial structuring of a decision problem in terms of selecting criteria or attributes and decision options. It is in this sense that the adjectives multi-criteria and multi-attribute analysis are used interchangeably in the literature (DCLG, 2009).

A typical MCDA approach evolves along three stages:

- structuring the decision problem;

- modeling the stakeholder preferences; and

- ranking the alternative choices.

While there are many MCDA methods available for solving a decision problem, there is no single MCDA method that has be proven superior for all decision-making problems. In general, different methods lead to different decisions but it has been argued that the ranking of alternative choices is method-independent provided that data are handled "appropriately" (Guitouni and Martel 1998).

Selecting an appropriate MCDA method based on criteria in the literature, problem constraints and the bias of the user is a multi-criteria problem in its own right. There are no clear guidelines on how to choose an appropriate MCDA method but the prevailing view remains that the simpler method should be chosen in any given circumstance (Guitouni and Martel 1998).

There is of course a gap between the theoretical power of MCDA methods and their applicability to real-world problems. This gap is not trivial when the problems are not very well understood and the constraints are ill defined. Traditional MCDA techniques based on operations research principles are often incapable of handling qualitative information and collapse when numerical data is lacking and imprecise.

\section{Mixed Mode Analysis}

In real-world situations, decision analysis is usually a prospective evaluation tool for the examination of alternative choices for a policy or an intervention. It is used that is to forecast in advance the intended results of a 
particular action or series of actions. In retrospective evaluations, decision analysis can contribute to the assessment of an implemented policy or intervention through the appraisal of its impact with regard to pre-determined criteria. In both cases, multi-criteria analysis is undertaken to integrate into a coherent framework quantitative and qualitative information, long-time intervention horizons, decision-making uncertainties and heterogeneous criteria.

A characteristic example of such mixed-mode decision analysis is the recent requirement to consider sustainability dimensions of economic, environmental or social interventions. There is a distinct need to develop a framework capable of handling qualitative information and missing or imprecise numerical data. Such an integrative framework should have the potential to provide well-structured, defensible and transparent solutions to complex decision problems.

Traditional MCDA techniques, deeply-rooted in the (mostly) deterministic world of operations research collapse under such circumstances. It is impossible to design interventions in fields such as regional development when the time-span for the collection of reliable data and the cost of sophisticated analysis often exceeds the timescales and budgets set aside for a policy assessment. There are practical difficulties in choosing the interventions to be studied, in determining the relevant criteria, and in addressing the lack of reliable data over a period of time sufficient to validate the methodologies employed. Furthermore, the stakeholders should be skilled in mathematical concepts and data aggregation techniques to appreciate the iterative nature of the processes and the nuisances of the proposed solutions. Often the stakeholders feel that multi-criteria analysis is a subjective tool used to advance pre-determined agendas (Montibeller and Franco 2010).

In such challenging situations, where there is still a need to pass judgment on complex interventions, multi-criteria analysis should use simple methodologies, limited to the comparison of a small set of activities through a set of finite criteria set (or easily understood) by the stakeholders.

In this modern milieu, the International Association for Impact Assessment (2013) defines multi-criteria analysis as follows:

«Multi-Criteria Decision Analysis (MCDA) is a general term for systematic and transparent approaches to analyze complex problems involving multiple criteria. We consider the term MCDA to also cover simple structuring methods and tools such as objective hierarchies, strategy tables, consequence tables and (conceptual) influence diagrams. The systematic MCDA framework can contribute the assessments by integrating diverse information 
from scientists, experts and local people, and by incorporating the subjective preferences of stakeholders into the analysis.»

The definition of multi-criteria analysis of IAIA emerged to cover modern problems with fuzzy data and simultaneous use of quantitative and qualitative variables. These problems cannot be addressed by classical methods of operations research that demand quantification and deterministic accuracy (Munasinghe 2007). Mixed-mode multi-criteria analysis methods can effectively support the "structuring, assessment and decision making on complex policy issues" as was defined in the EU-funded LIAISE Network of Excellence on impact assessment research for sustainable development (Geneletti, 2013).

The sentiment is echoed in the World Bank (2000) manual on MultiCriteria Analysis for development:

«Multi-Criteria Analysis techniques aim to provide a rational basis for classifying choice through the aggregation of disparate information onto a common index of utility or value. Multi-criteria techniques encompass a large family of methods of which 40 or more different approaches are distinguishable in the literature, from the highly sophisticated through to simple rating systems.» and the claimed objective for techniques that have the capacity to simplify complex situations.

\section{GE/McKinsey Matrices}

The most basic requirement in modern multi-criteria analysis is to undertake calculations and aggregations that yield performance tables for each intervention, based on carefully weighted criteria that measure their relative importance for each stakeholder. These performance tables should be able to accommodate mixed-mode information and be relatively immune to data noise.

In this context GE/McKinsey matrices, an old stalwart of business portfolio analysis, have received renewed attention and were successfully employed in the MCDA assessment of business cluster policies (Tsakalerou 2014).

The GE/McKinsey Matrices are typically nine-cell (3X3) matrices used to perform portfolio analysis in the strategic planning process of an enterprise. They were invented by General Electric in the 1970s and perfected by McKinsey later on. In essence, GE/McKinsey Matrices are a multi-factorial analysis technique used in product management to help a company decide what product(s) to add to its portfolio.

Typically, one dimension of the matrices comprises three industry attractiveness levels (low, medium and high) while the other comprises three internal business strength measures (low, medium and high). Each product, 
brand, service, or potential product is mapped in this industry attractiveness/business strength space (Coyne 2008) with three distinct decisions possible: invest/grow, selectively change and harvest/divest. GE/McKinsey Matrices differ from similar tools, like the Boston Consulting Group Matrix, in that multiple factors are used to define industry attractiveness and business unit strength.

Grouping the variables of a problem and their effect on performance in three classes is the major advantage of the GE/McKinsey matrices in identifying central trends in the data. Indeed, GE/McKinsey matrices exploit a fundamental concept in statistics, namely that choosing a small (and preferably odd) number of classes for grouping the data is one effective way to reduce the impact of noise in the data (Newbold, Carlson and Thorne 2009). The situation is akin to low-pass filtering in signals, where the processing of noisy waveforms with low-pass filters is used to improve the signal-to-noise ratio (Smith 2002).

The GE/McKinsey variable-pair analysis can be employed in a totally different context, namely to assess variable performance in multivariable modeling problems complicated by the need to integrate diverse information and imprecise data.

The applicability of GE/McKinsey matrices in organizing and presenting the results of large multi-criteria studies has been demonstrated persuasively (Tsakalerou 2014). In the context of assessing the effect of intellectual capital on firm performance, a modified version of the GE/McKinsey matrices was used to satisfy the quest for appropriate mixedmode multi-criteria analysis methods. This practical use of the GE/McKinsey matrices exemplified their utility in integrating mixed-mode data (ordinal, categorical and semi-quantitative) in the same decision framework.

Specifically, the following adaptation (mode) of the GE/McKinsey Matrices was proposed for variable-pair analysis:

\begin{tabular}{|c|c|c|c|c|}
\hline \multirow{3}{*}{ 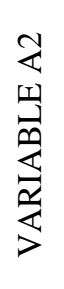 } & High & $\mathrm{V}_{31}$ & $V_{32}$ & $\mathrm{~V}_{33}$ \\
\hline & Medium & $\mathrm{V}_{21}$ & $\mathrm{~V}_{22}$ & $\mathrm{~V}_{23}$ \\
\hline & Low & $\mathrm{V}_{11}$ & $V_{12}$ & $\mathrm{~V}_{13}$ \\
\hline \multirow{2}{*}{\multicolumn{2}{|c|}{$\begin{array}{l}\text { GE/McKINSEY } \\
\text { MATRIX MODE }\end{array}$}} & Low & Medium & High \\
\hline & & \multicolumn{3}{|c|}{ VARIABLE A1 } \\
\hline
\end{tabular}


Each block (i,j) in the matrix is assigned a weighting $\mathrm{V}_{\mathrm{ij}}$ appropriate for the situation. In this sense, the entries are calculated as follows:

$\mathrm{V}_{\mathrm{ij}}=\mathrm{SUM}$ [Cases in $(\mathrm{i}, \mathrm{j}) \mathrm{x}$ Quantified Effect] / SUM [Cases in $\left.(\mathrm{i}, \mathrm{j})\right]$

or

$\mathrm{V}_{\mathrm{ij}}=\mathrm{SUM}$ [Firms in $(\mathrm{i}, \mathrm{j}) \mathrm{x}$ Quantified Effect] / SUM [Firms in (i,j)] thus enabling comparisons between the pair of variables. The weights $V_{i j}$ are in effect the weighted averages of the effect on performance for all the cases (or firms) in the dataset that satisfy the $(\mathrm{i}, \mathrm{j})$ criteria with respect to the variables $\mathrm{A}_{1}$ and $\mathrm{A}_{2}$.

If the grouping of the variables in three classes is accompanied by rounding-up the calculations the impact of imprecisions in the data is further diminished. In this sense, only major trends are identified, a fact which strengthens the utility of the conclusions of the analysis. In doing so, the use of an appropriately modified form of GE/McKinsey matrices emerges naturally as an effective mixed-mode tool of choice.

\section{Conclusions}

Multi-criteria analysis has evolved from its operations research origins to address situations where the criteria are not so easily quantified and where the data is often fuzzy. In such challenging situations, where there is a need to assess complex interventions, multi-criteria analysis should use simple methodologies, limited to a small set of activities and a well-defined set of finite criteria set.

In this context, GE/McKinsey matrices, a classic tool of the business portfolio analysis, have been demonstrated to be an effective tool to address mixed-mode problems. By grouping decision variables and their effects into a small number of classes, GE/McKinsey matrices provide an effective way to reduce the effect of noise in the data and to allow for major trends to emerge. Thus GE/McKinsey matrices rightly belong to the arsenal of modern MCDA techniques.

\section{References:}

Belton, S. \& Stewart, T.S., (2002) Multiple Criteria Decision Analysis. An Integrated Approach. Kluwer, Boston.

Coyne, K. (2008) "Enduring Ideas: the GE-McKinsey nine-box matrix". McKinsey Quarterly. September issue.

Department for Communities and Local Government (2009) Multi-criteria analysis: A Manual. London. 
Geneletti, D. (2013) Multi-criteria analysis. LIAISE Toolbox. Retrieved from: beta.liaise-toolbox.eu/ia-methods/multi-criteria-analysis.

Guitouni, A. \& Martel, J.M. (1998) "Tentative guidelines to help choosing an appropriate MCDA method". European Journal of Operations Research, 109:501-521.

Harris R (1998) Introduction to decision making. Virtual Salt. Retrieved from: www.virtualsalt.com/crebook.htm.

IAIA (2013) "Impact Assessment - the Next Generation". In Proceedings of the 33rd Annual Meeting of the IAIA, Calgary, Alberta.

Lu, J., Zhang, G., Ruan, D., \& Wu, F. (2007) Multi-objective Group Decision Making. Imperial College, London.

Montibeller, G. \& Franco, A. (2010) "Multi-Criteria Decision Analysis for Strategic Decision Making", in the Handbook of Multicriteria Analysis. Springer, Berlin, 2010.

Munasinghe, M. (2007) "Multi-criteria analysis in environmental decisionmaking". Encyclopedia of Earth.

Newbold, P., Carlson, W., \& Thorne, B. (2009). Statistics for Business and Economics (7th ed.). Pearson Education.

Smith, S.W. (2002) Digital Signal Processing: A Practical Guide for Engineers and Scientists. CalTech Publishing, San Diego.

Tsakalerou, M. (2014) Business Clusters in the Service Sector: Multicriteria Analysis and Modeling. $\mathrm{PhD}$ dissertation, Department of Production Engineering and Management, Democritus University of Thrace, Xanthi, Greece.

World Bank (2000) Multi-Criteria Analysis. Environmental Resources Management. Online at: www.worldbank.org/urban/solid_wm/erm/. 